\title{
STOCHASTIC BEHAVIOUR IN SUB-PLY FAILURE MECHANISM OF UNIDIRECTIONAL CARBON FIBER COMPOSITES
}

\author{
$\underline{\text { D. Garoz }}^{1^{*}}$, M. Flores ${ }^{2}$, D. Mollenhauer ${ }^{2}$, C. Gonzalez ${ }^{1}$ \\ ${ }^{1}$ IMDEA Materials, C/Eric Kandel 2, 28906 Getafe, Madrid, Spain \\ ${ }^{2}$ U.S. Air Force Research Laboratory, 2941 Hobson Way, WPAFB, OH 45433-7750, USA \\ *david.garoz@imdea.org
}

The sub-ply failure mechanism of a fibre-reinforced composite laminate is the key to understand and prevent the failure of composite structures under compressive loads. Fibrematrix debonding and matrix cracking occur in the sub-ply failure mechanism when the load is transverse to the fibres. These two damage modes trigger a stochastic damage progression through the ply.

The sub-ply failure mechanism of unidirectional carbon fibre composites has been studied using finite element simulations for the case of a micro-pillar specimen under compression load. The dimensions and the volume fraction determine the geometry of the micro-pillar. The model describes the sub-ply failure under the specified compression load based on the mechanical properties of the constituents, fibre and matrix as well as their interface. Then, the stochastic behaviour of the failure mechanism has been investigated considering different random geometries, the variability of the mechanical properties, and heterogeneous distributions of defects. Figure 1 shows the final failure of two different micro-pillar geometries keeping the volume fraction and material properties. Although the failure patterns are different, the stressstrain curves show a general behaviour with a limited dispersion of the maximum strength. As a preliminary conclusion, the proposed model can determine the dispersion of the relevant mechanical properties for the sub-ply failure under compression considering different sources that affect the failures mechanism. Finally, the simulated sub-ply failures are compared to dedicated experiments of micro-pillars performed under a SEM microscope.

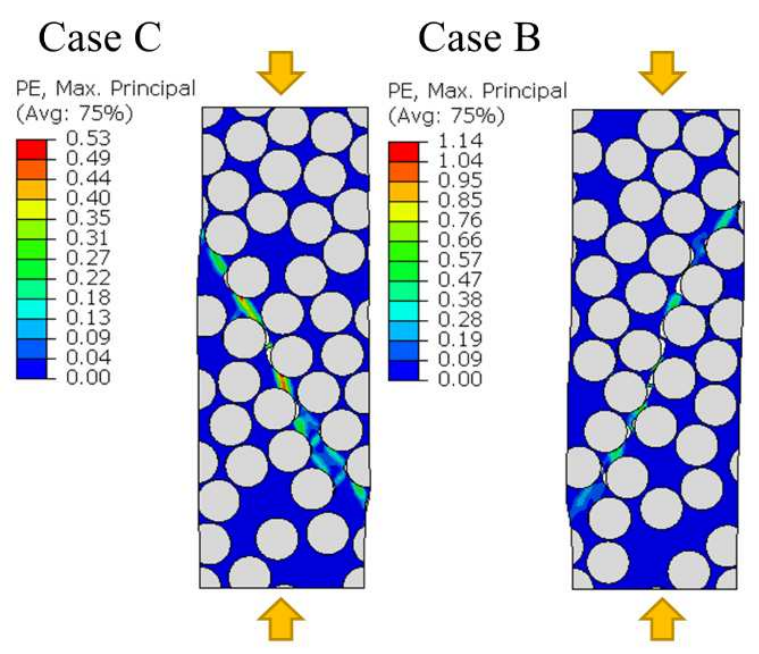

(a) Micro-pillars under compression

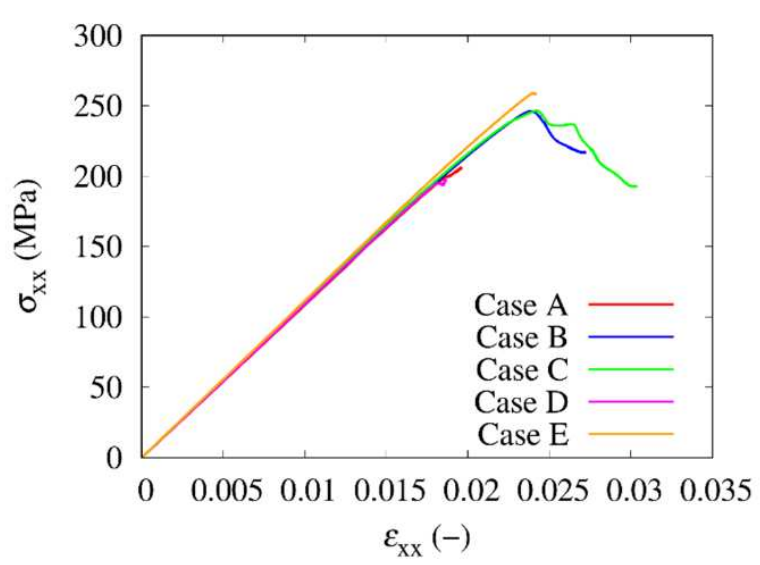

(b) Stress-strain behaviour

Figure 1. (a) Maximum principal plastic strain field of two micro-pillar under compression, and (b) the stress-strain behaviour of different micro-pillars with same dimensions and volume fraction. 